\title{
Secreted PCSK9 decreases the number of LDL receptors in hepatocytes and in livers of parabiotic mice
}

Thomas A. Lagace, ${ }^{1}$ David E. Curtis, ${ }^{2}$ Rita Garuti, ${ }^{1}$ Markey C. McNutt, ${ }^{1}$ Sahng Wook Park, Heidi B. Prather, ${ }^{1}$ Norma N. Anderson, ${ }^{1}$ Y.K. Ho, ${ }^{1}$ Robert E. Hammer, ${ }^{3}$ and Jay D. Horton ${ }^{1,4}$

\begin{abstract}
1Department of Molecular Genetics, ${ }^{2}$ Department of Surgery, ${ }^{3}$ Department of Biochemistry, and ${ }^{4}$ Department of Internal Medicine,
\end{abstract} University of Texas Southwestern Medical Center, Dallas, Texas, USA.

\begin{abstract}
Proprotein convertase subtilisin/kexin type 9 (PCSK9) is a member of the proteinase K subfamily of subtilases that reduces the number of LDL receptors (LDLRs) in liver through an undefined posttranscriptional mechanism. We show that purified PCSK9 added to the medium of HepG2 cells reduces the number of cellsurface LDLRs in a dose- and time-dependent manner. This activity was approximately 10-fold greater for a gain-of-function mutant, PCSK9(D374Y), that causes hypercholesterolemia. Binding and uptake of PCSK9 were largely dependent on the presence of LDLRs. Coimmunoprecipitation and ligand blotting studies indicated that PCSK9 and LDLR directly associate; both proteins colocalized to late endocytic compartments. Purified PCSK9 had no effect on cell-surface LDLRs in hepatocytes lacking autosomal recessive hypercholesterolemia (ARH), an adaptor protein required for endocytosis of the receptor. Transgenic mice overexpressing human PCSK9 in liver secreted large amounts of the protein into plasma, which increased plasma LDL cholesterol concentrations to levels similar to those of LDLR-knockout mice. To determine whether PCSK9 was active in plasma, transgenic PCSK9 mice were parabiosed with wild-type littermates. After parabiosis, secreted PCSK9 was transferred to the circulation of wild-type mice and reduced the number of hepatic LDLRs to nearly undetectable levels. We conclude that secreted PCSK9 associates with the LDLR and reduces hepatic LDLR protein levels.
\end{abstract}

\section{Introduction}

Proprotein convertase subtilisin/kexin type 9 (PCSK9) is a member of the proteinase K subfamily of subtilisin-related serine endoproteases. Like other members of this family, the PCSK9 protein has a signal sequence, followed by a prodomain, and a catalytic domain that contains a conserved triad of residues (D186, H226, and S386) required for activity (1). PCSK9 is synthesized as a soluble, approximately $74-\mathrm{kDa}$ precursor that undergoes intramolecular autocatalytic cleavage in the ER, generating a $14-\mathrm{kDa}$ prodomain and an approximately $60-\mathrm{kDa}$ catalytic fragment. The site of intramolecular cleavage (VFAQ $\downarrow$ SIP) differs from that of subtilisin and other family members that typically cleave after a basic residue $(2,3)$. The cleaved prodomain remains associated with the catalytic domain, forming a PCSK9-prodomain complex that is transported to the Golgi complex, where it undergoes sulfation prior to secretion $(1,3)$.

Insights into the physiological function of PCSK9 initially came from the identification of point mutations in PCSK9 that cause an autosomal dominant form of hypercholesterolemia (4). These mutations were later shown to be gain-of-function alleles that act in a dominant fashion $(3,5)$. PCSK9 was independently identified as a SREBP-regulated gene in liver, using microarrays hybridized with RNA from livers of mice that either overexpressed or lacked

Nonstandard abbreviations used: ARH, autosomal recessive hypercholesterolemia; CI-MPR, cation-independent mannose-6-phosphate receptor; LDLR, LDL receptor; LRP, LDLR-related protein; MEF, mouse embryonic fibroblast; PCSK9, proprotein convertase subtilisin/kexin type 9 .

Conflict of interest: J.D. Horton is a consultant for Alnylam Pharmaceuticals, Aegerion Pharmaceuticals Inc., Metabasis, Merck, and Pfizer, and is on the Speakers' Bureau of Merck, Merck/Schering-Plough Pharmaceuticals, Schering-Plough. Citation for this article: J. Clin. Invest. 116:2995-3005 (2006). doi:10.1172/JCI29383.
SREBPs $(6,7)$. SREBPs are a family of transcription factors that increase the expression of many genes involved in cholesterol and fatty acid synthesis, as well as the $L D L$ receptor $(L D L R)$ gene (6). The induction of PCSK9 by SREBPs further suggested that this protein was involved in lipid metabolism.

The biological activity of PCSK9 was revealed through overexpression studies in mice. Overexpression of PCSK9 posttranscriptionally reduced the amount of LDLR protein in liver $(3,8-10)$. Confirmation that PCSK9 functions normally to regulate LDLR protein levels came from loss-of-function studies in humans and mice. Individuals who are heterozygous for a nonsense mutation in allele PCSK9 have significantly lower plasma LDL cholesterol levels, suggesting that a reduction in PCSK9 activity leads to an increase in LDLRs (11). These conclusions were supported by studies in PCSK9-knockout mice, which revealed that loss of PCSK9 resulted in increased numbers of LDLRs in hepatocytes, accelerated plasma LDL clearance, and significantly lower plasma cholesterol levels (12). In the most recent studies, humans heterozygous for loss-of-function mutations in PCSK9 were shown to have a significant reduction in the long-term risk of developing atherosclerotic heart disease (13).

The genetic data from humans and the in vivo studies in mice demonstrate that one function of PCSK9 is to reduce the number of the LDLRs and that this function is manifest in humans in the basal state. The mechanism by which PCSK9 reduces the number of LDLRs is still undetermined. For example, it is unclear whether PCSK9 acts to destroy LDLRs in the secretory pathway or whether it acts outside of the cell. In the current studies, we provide evidence that extracellular PCSK9 can be internalized by cultured liver cells and fibroblasts in a manner that is largely dependent on LDLRs. Incubation with extracellular PCSK9 led to loss of LDLRs 


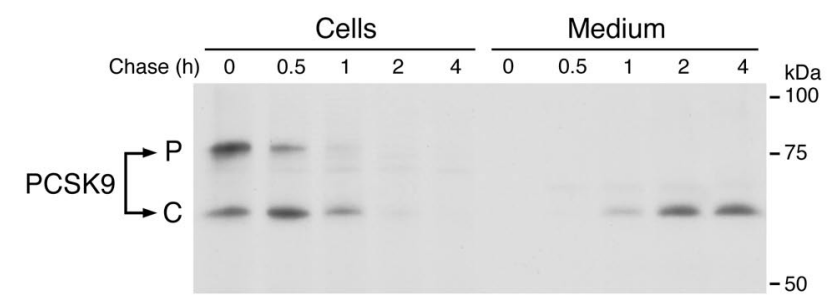

Figure 1

Pulse-chase analysis of endogenous PCSK9 secretion from HepG2 cells. Cells were cultured in sterol-depleting medium $C$ for 18 hours prior to labeling for 30 minutes with $\left[{ }^{35} \mathrm{~S}\right]$ methionine/cysteine. After washing, cells were incubated in chase medium containing unlabeled methionine and cysteine for the indicated times. Cells were lysed, and PCSK9 was immunoprecipitated from the medium and cell lysates as described in Methods. Samples were subjected to $8 \%$ SDS-PAGE, and the gel was treated with Amplify fluorogenic reagent prior to drying and exposure to film. $P$ and $C$ denote the proprotein and cleaved forms of PCSK9, respectively. Similar results were obtained in 4 independent experiments.

from the cell surface and accelerated destruction of LDLRs in liverderived cells. Finally, we demonstrate that increased PCSK9 levels in the circulation of mice leads to diminished liver LDLR protein and increased plasma cholesterol levels.

\section{Results}

PCSK9, like other subtilisin family proteases, is synthesized with a prodomain $(1,3)$. In the other subtilisin family proteases, the cleaved prodomain remains associated with the protein and acts as an inhibitor of the cognate enzyme activity. The prosegment often undergoes secondary proteolytic processing, which relieves this inhibition and unmasks enzymatic activity (14). When PCSK9 is overexpressed in cultured cells, the protein is secreted with the prodomain still attached $(1,3)$. This result raised the possibility that cellular PCSK9 remains inactive within the secretory pathway and that PCSK9 may act on LDLRs after secretion, either at the cell surface or after the LDLR is internalized into the endosomal/lysosomal system.

To test this possibility, we assessed the rate and extent of secretion of endogenous PCSK9 expressed at physiological levels in cultured human hepatoma HepG2 cells. This study was made possible by the development of antibodies against the catalytic domain of PCSK9. Synthesis and secretion of PCSK9

\section{Figure 2}

Reduction of endogenous LDLRs in HepG2 cells following the addition of recombinant purified PCSK9 to the culture medium. (A) Dose response of exogenous PCSK9-mediated LDLR degradation in HepG2 cells. Cells were cultured for 18 hours in medium C prior to treatment for 4 hours with the indicated amounts of purified human PCSK9. (B) Time course of exogenous PCSK9-mediated LDLR degradation. HepG2 cells cultured as described above were treated with $5 \mu \mathrm{g} / \mathrm{ml}$ of purified PCSK9 for the indicated times. Total lysates were prepared following cell-surface biotinylation as described in Methods. Proteins were subjected to SDS-PAGE for immunoblot analysis of the LDLR using IgG-HL1 and anti-FLAG M2 monoclonal antibody to detect purified PCSK9. The transferrin receptor protein was detected as described in Methods and used as a control for loading and nonspecific protein degradation. Similar results were obtained in 3 independent experiments. were quantified by pulse-labeling the cells with $\left[{ }^{35} \mathrm{~S}\right]$ methionine/ cysteine, which was followed by a chase period in isotope-free medium. Radiolabeled PCSK9 was immunoprecipitated from cells and the medium at different times. Figure 1 shows results of a representative pulse-chase experiment. Two forms of the protein, corresponding to the uncleaved precursor $(\sim 74 \mathrm{kDa})$ and the cleaved mature catalytic fragment $(\sim 60 \mathrm{kDa})$, were detected in cells at 0.5 and 1 hour of chase. After 2 hours, none of the labeled uncleaved PCSK9 was detected in the cells. After 4 hours, nearly all of the radiolabeled PCSK9 was recovered in the medium, demonstrating that the cleaved form of PCSK9 is rapidly and efficiently secreted from these cells.

We recently reported that PCSK9 can be detected in human plasma by immunoprecipitation (15). To quantify the concentration and determine the physiological range of PCSK9 in human plasma, we developed an ELISA that used an anti-human PCSK9 monoclonal antibody to capture PCSK9 and a polyclonal antihuman PCSK9 antibody for detection. Plasma levels of PCSK9 were quantified in 72 volunteers. The plasma levels ranged from approximately 50 to approximately $600 \mathrm{ng} / \mathrm{ml}$ (Supplemental Figure 1; supplemental material available online with this article; doi:10.1172/JCI29383DS1). These measurements demonstrate that considerable amounts of PCSK9 circulate in plasma and provided a range of physiologically relevant PCSK9 concentrations.

We next determined whether the secreted form of PCSK9 can reduce the number of LDLRs when added to the medium of HepG 2 cells cultured in sterol-depleted medium to induce LDLR expression. Recombinant PCSK9 that contained a FLAG tag at the carboxyl terminus was purified from HEK 293S cells as described in Methods. SDS-PAGE and Coomassie blue staining analysis showed that purified FLAG-tagged PCSK9 consisted of the catalytic fragment and the cleaved prodomain that migrated with apparent molecular masses of approximately $60 \mathrm{kDa}$ and approximately $17 \mathrm{kDa}$, respectively (Supplemental Figure 2A). Gel filtra-

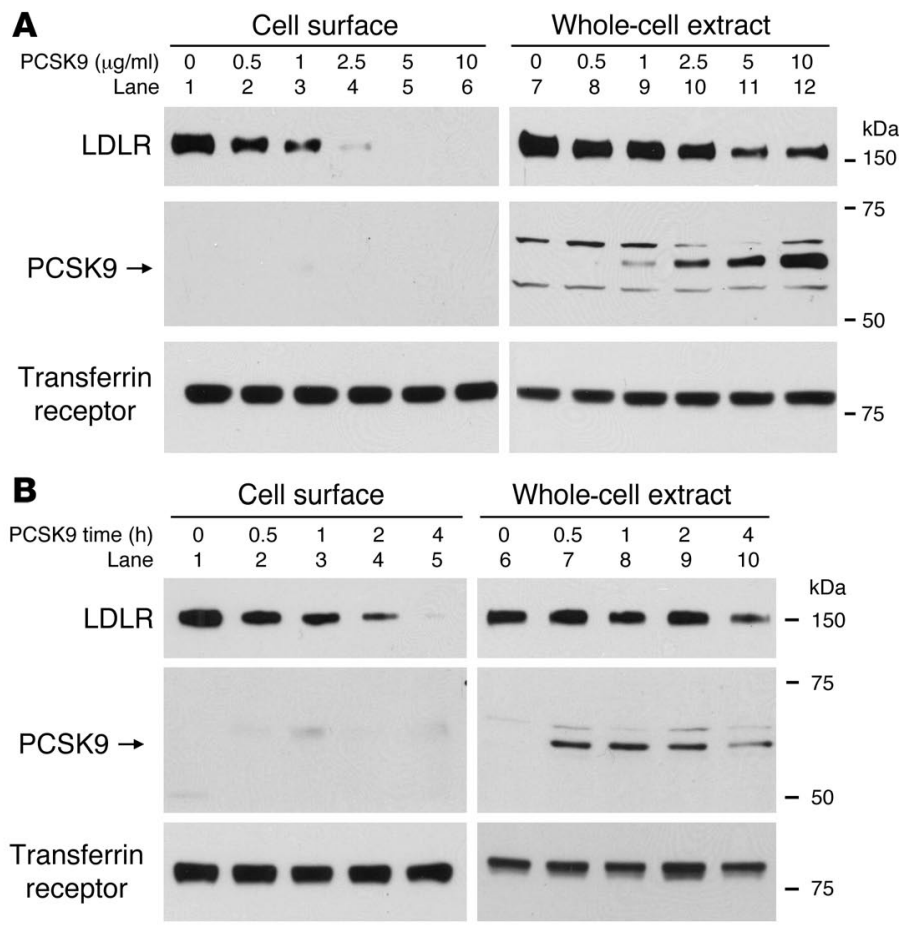




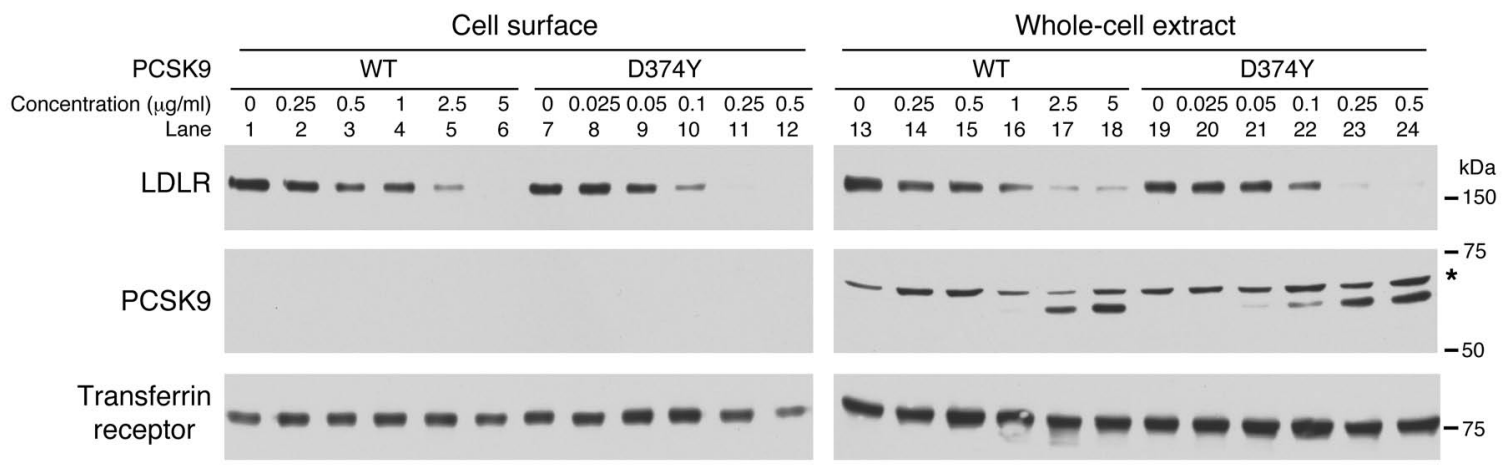

Figure 3

Increased cell association and LDLR degradation by addition of purified mutant PCSK9(D374Y) to the medium of HepG2 cells. Cells were cultured for 18 hours in medium C and then incubated for 4 hours with the indicated amounts of purified human PCSK9 or PCSK9(D374Y). Immunoblot analysis of LDLR, FLAG-tagged PCSK9, and transferrin receptor was carried out as described in the legend to Figure 2 . The asterisk indicates a nonspecific band. Similar results were obtained in 3 independent experiments.

tion revealed that the protein elutes at a single peak volume corresponding to an apparent molecular mass of approximately $70 \mathrm{kDa}$ (Supplemental Figure 2B). After incubation with purified PCSK9, the surface proteins of HepG2 cells were covalently modified with a cell-impermeable biotinylation reagent and isolated using streptavidin beads. The total cellular LDLRs in whole-cell extracts and cell-surface LDLRs were measured by SDS-PAGE immunoblotting using a monoclonal antibody. The total cell PCSK9 accumulation and cell-surface PCSK9 were also measured by SDS-PAGE and immunoblotting, but with anti-FLAG antibody.

As shown in Figure 2A, the number of cell-surface LDLRs declined by approximately $50 \%$ after incubation with the physiologically relevant concentration of $0.5 \mu \mathrm{g} / \mathrm{ml}$ PCSK9 (lane 2) and became nearly undetectable after exposure to $2.5 \mu \mathrm{g} / \mathrm{ml}$ PCSK9 (lane 4). Incubation of HepG2 cells for 4 hours with 5 or $10 \mu \mathrm{g} / \mathrm{ml}$ PCSK9 decreased whole-cell LDLR protein levels by approximately 50\% (lanes 11 and 12). FLAG-tagged PCSK9 was detected in wholecell extracts in a concentration-dependent manner (lanes 7-12), but it was not detected among the biotin-labeled cell-surface proteins (lanes 1-6), suggesting that most of the cell-associated PCSK9 had been internalized.

To determine the time course of PCSK9 action, HepG2 cells were incubated in the presence of $5 \mu \mathrm{g} / \mathrm{ml}$ PCSK9 and then harvested at different intervals over a 4-hour period (Figure 2B). The.number of cell-surface LDLRs declined noticeably at 2 hours (lane 4) and was undetectable at 4 hours (lane 5). The amount of cell-associated FLAG-tagged PCSK9 was maximal after 30 minutes (lane 7) and decreased slightly at 4 hours (lane 10). PCSK9 had no effect on whole-cell or cell-surface transferrin receptors (Figure 2, A and B).

As discussed in the Introduction, certain point mutations in PCSK9 cause hypercholesterolemia. To determine whether one such mutation increases the activity of PCSK9 in a cell-based assay, varying amounts of wild-type PCSK9 and the PCSK9 mutant D374Y (4) were added to HepG2 cells, after which LDLR protein levels were measured (Figure 3 ). The D374Y mutation was chosen for study because individuals who harbor this mutation have been shown to manifest severe hypercholesterolemia (16). PCSK9(D374Y) was at least 10-fold more active than wild-type PCSK9 in reducing cell surface LDLRs. Thus, PCSK9(D374Y) at $0.25 \mu \mathrm{g} / \mathrm{ml}$ was at least as effective as $2.5 \mu \mathrm{g} / \mathrm{ml}$ of wild-type PCSK9 (compare lanes 5 and 11). After incubation with wild-type PCSK9, the number of LDLRs was significantly reduced in whole-cell extracts, and similar results were obtained with 10-fold lower concentrations of PCSK9(D374Y) (lanes 13-24). Despite the different concentrations employed, the amounts of wild-type and mutant PCSK9 measured in the cell extracts were similar, indicating that the mutant protein was taken up by the cell approximately 10 -fold more efficiently than the wild-type protein.

To determine whether cellular association/uptake of PCSK9 is dependent upon LDLRs, we turned to mouse embryonic fibroblasts (MEFs), to exploit the availability of cells from gene knockout mice that lack the LDLR and the closely related receptor, the LDLR-related protein (LRP). MEFs derived from wild-type, $\mathrm{Ldlr}^{-1}$, $\mathrm{Lrp}^{-/-}$, and $\mathrm{Ldlr} \mathrm{r}^{--} \mathrm{Lrp}^{-/-}$mice were incubated with varying amounts of PCSK9 (Figure 4A). Immunoblots revealed that abundant PCSK9 was associated with the wild-type (lanes 1-4) and $\mathrm{Lrp}^{-/-}$cells (lanes 9-12). PCSK9 cellular association was markedly reduced in $\mathrm{Ldlr}^{/-}$MEFs (lanes 5-8); however, a small amount of PCSK9 was detectable in cells incubated with the highest concentration of PCSK9 (lane 8). This small amount of association was abolished in cells that lacked both LDLR and LRP (lane 16). These data suggest that exogenous PCSK9 associates with MEFs in a manner that is almost totally dependent upon the LDLR and that LRP may play a small role in uptake at high concentrations of PCSK9.

The uptake of PCSK9 was characterized morphologically using indirect immunofluorescence in MEFs. Double immunofluorescence labeling of cells incubated with PCSK9 for 4 hours showed diffuse localization of the protein in small punctate structures, possibly representing endocytic vesicles, which partially overlapped with the LDLR staining pattern (Figure 4B). To determine whether these structures were endosomal and whether PCSK9 colocalized intracellularly with the LDLR, we incubated MEFs in the presence of chloroquine, which raises the $\mathrm{pH}$ of acidic cellular compartments and inhibits lysosomal hydrolases. In the presence of chloroquine, the cation-independent mannose-6phosphate receptor (CI-MPR) accumulates in late endosomes and was used as a marker of these structures (17). As shown in Figure 4C, PCSK9 became concentrated in large perinuclear vacuoles of MEFs in the presence of chloroquine. These vacuoles were also labeled with anti-LDLR and anti-CI-MPR antibodies. Considered together, the data indicate that exogenous PCSK9 is taken up by fibroblasts in a manner that is dependent upon the LDLR and 

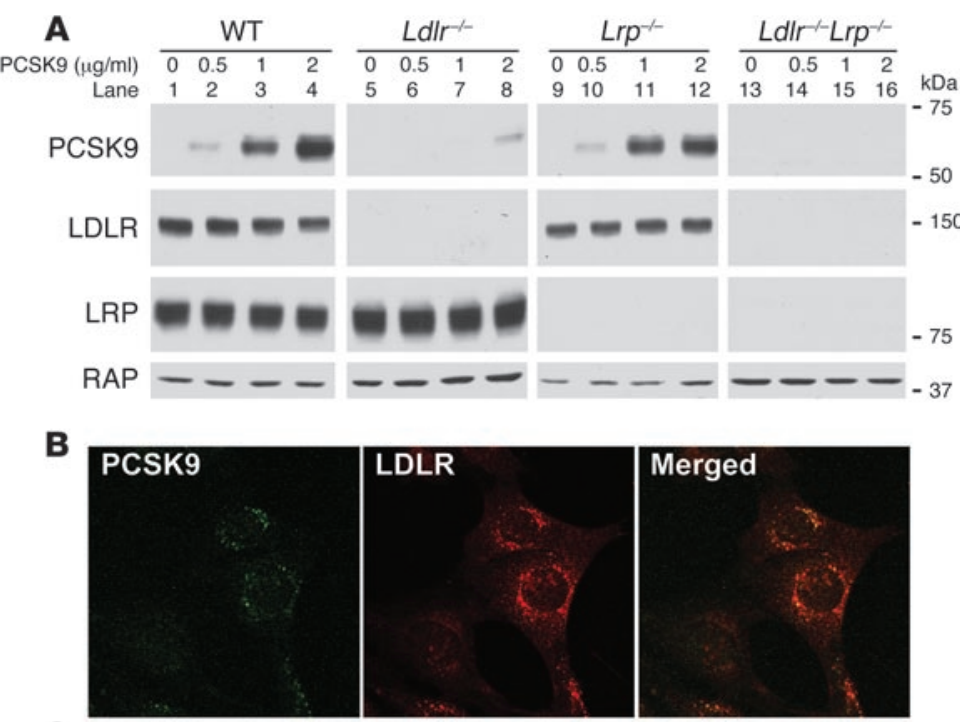

C
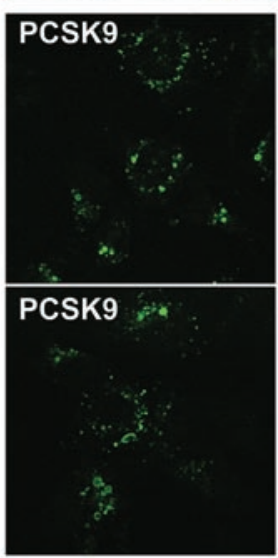
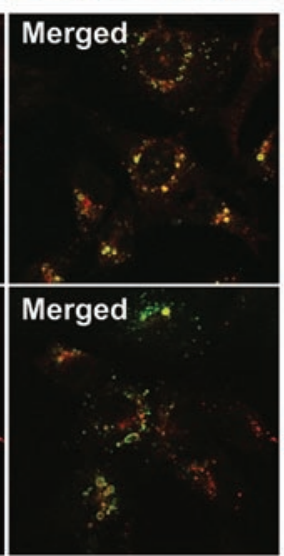

\section{Figure 4}

LDLR-dependent endocytosis of PCSK9 in MEFs. (A) Immunoblot analysis of PCSK9 association with MEFs. Immortalized MEFs derived from wild-type, $\mathrm{LdIr}^{-1-}, \mathrm{Lrp}^{-/-}$, and $L d l r^{--} \mathrm{Lrp}^{-/-}$mice were cultured for 18 hours in medium F prior to treatment with purified human PCSK9 for 4 hours. Cell lysates $(30 \mu \mathrm{g})$ were subjected to SDS-PAGE and immunoblot analysis using IgG-15A6 to detect PCSK9 or a polyclonal antiserum (Ab 3143) that recognizes the LDLR, as described in Methods. Immunoblots of receptorassociated protein (RAP) were used as a loading control. (B) Indirect immunofluorescence localization of PCSK9 and the LDLR in wild-type MEFs. MEFs were incubated for 4 hours with $5 \mu \mathrm{g} / \mathrm{ml}$ purified human PCSK9 and processed for double immunofluorescence of PCSK9 (green) and the LDLR (red) as described in Methods. (C) Indirect immunofluorescence localization of PCSK9, LDLR, and the late-endosomal marker Cl-MPR in MEFs cultured in the presence of chloroquine. Wild-type MEFs were incubated for 4 hours with $5 \mu \mathrm{g} / \mathrm{ml}$ purified human PCSK9 in the presence of $0.1 \mathrm{mM}$ chloroquine and processed for double immunofluorescence of PCSK9 (green) and the LDLR (red) or Cl-MPR (red) as described in Methods. The merged image shows areas of colocalization of PCSK9 with the LDLR and CI-MPR. Magnification, $\times 630$. Similar results were obtained in 3 independent experiments. that the PCSK9 travels with the LDLR to the endosome/lysosome. Despite this uptake, PCSK9 does not destroy the LDLR in fibroblasts at the concentrations tested, either in the absence or presence of chloroquine. A similar lack of destruction was observed earlier when PCSK9 was overexpressed in fibroblasts and Chinese hamster ovary cells by transfection (9).

The requirement of LDLRs for PCSK9 internalization suggests that PCSK9 may bind to the LDLR. Moreover, PCSK9(D374Y) was taken up with higher efficiency than wild-type PCSK9, suggesting that the mutant protein may bind to the LDLR more avidly than wild-type PCSK9. To test this possibility, coimmunoprecipitation studies with wild-type PCSK9 or mutant PCSK9(D374Y) and the LDLR were carried out in HepG2 cells. The cells were incubated with wild-type and mutant PCSK9 proteins containing FLAG epitope tags. After a 1-hour incubation in the presence of chloroquine, cells were solubilized, and exogenously added PCSK9 was precipitated with an anti-FLAG antibody. As shown in Figure 5A (lanes 1 and 5), the FLAG antibody efficiently pulled down the wild-type and mutant PCSK9 from cell extracts, and the immunoprecipitation was reduced in the presence of competing FLAG octapeptide (lanes 2 and 6). In cells that were incubated with wild-type PCSK9, no detectable LDLR was coimmunoprecipitated with PCSK9. In contrast, a significant amount of LDLR was pulled down from cells incubated with PCSK9(D374Y) (lanes 1 and 5). When the LDLR antibody was used for the immunoprecipitation, a small, but consistently detectable amount of wild-type PCSK9 was coimmunoprecipitated. The LDLR antibody pulled down much larger amounts of PCSK9(D374Y) (lanes 3 and 7).

To determine whether the interaction of PCSK9 and the LDLR was direct, ligand blotting was performed using purified extracellular domain LDLR protein and PCSK9. First, we determined the migration of the purified LDLR under reduced and nonreduced conditions (Figure 5B). Next, we determined whether PCSK9 could bind to the extracellular domain of the LDLR in a specific manner. The reduced and nonreduced LDLR protein was resolved by SDS-PAGE and transferred to nitrocellulose. The filters were then incubated with $5 \mu \mathrm{g} / \mathrm{ml}$ of purified PCSK9 protein, and bound PCSK9 was visualized using the monoclonal antibody IgG-15A6. As shown in Figure 5C, PCSK9 bound to nonreduced LDLR protein but not to LDLRs that had been reduced. The binding of wild-type PCSK9 and PCSK9(D374Y) to increasing amounts of the LDLR protein was then determined by ligand blotting (Figure 5D). Both forms of purified PCSK9 bound to the extracellular domain of the LDLR protein in a concentrationdependent manner. Consistent with the coimmunoprecipitation, the PCSK9(D374Y) mutant appeared to bind to the LDLR protein with a greater affinity. Combined, the results of these studies indicate that PCSK9(D374Y) binds to LDLRs with higher affinity than does wild-type PCSK9, a finding that correlates with the enhanced ability of the mutant PCSK9 to destroy LDLRs. 
A
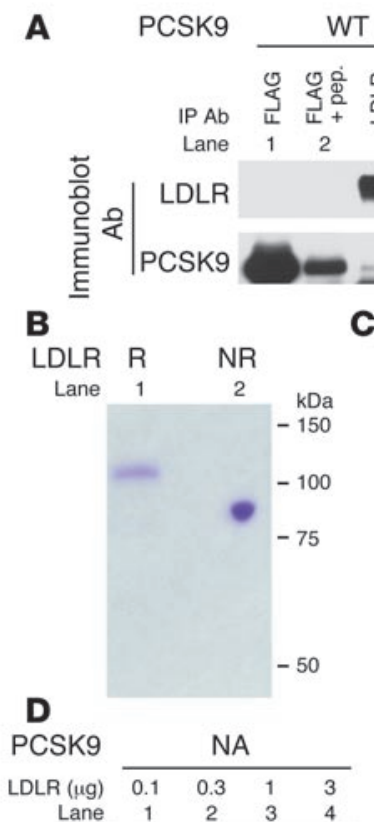

\section{WT}

\section{을}
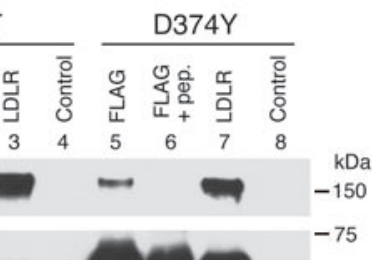

\section{$\mathrm{kDa}$}

$-75$
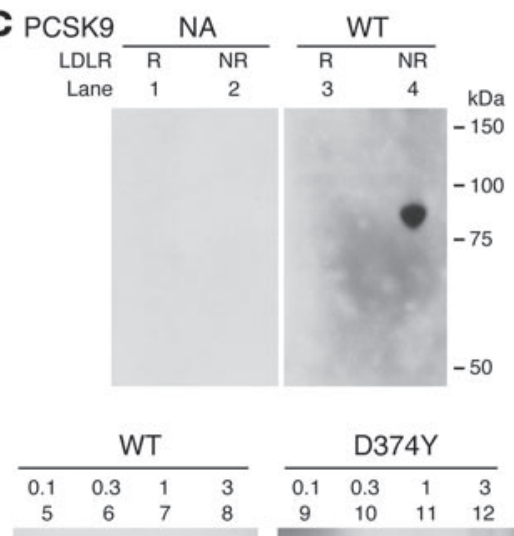
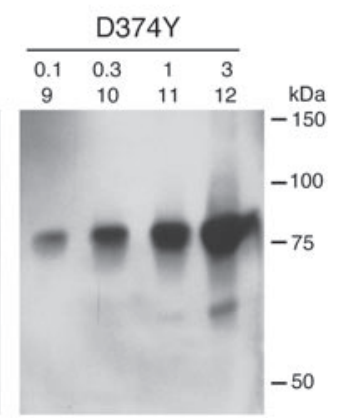

\section{Figure 5}

Association of PCSK9 and PCSK9(D374Y) with the LDLR. (A) Coimmunoprecipitation of the LDLR and exogenously added wild-type PCSK9 or PCSK9(D374Y) protein. HepG2 cells were cultured for 18 hours in medium $\mathrm{C}$ prior to treatment for 1 hour in the presence of $0.1 \mathrm{mM}$ chloroquine with $20 \mu \mathrm{g} / \mathrm{ml}$ or $2 \mu \mathrm{g} / \mathrm{ml}$ of purified human PCSK9 or PCSK9(D374Y), respectively. Cells were harvested and lysed and proteins immunoprecipitated with the indicated antibodies. Pellets of the immunoprecipitation were subjected to SDS-PAGE and immunoblot analysis. Polyclonal antiserum (Ab 3143) was used to detect LDLR, and IgG-15A6 was used to detect PCSK9. (B) Reduced (R) or nonreduced (NR) LDLR extracellular domain protein $(2 \mu \mathrm{g})$ was resolved by SDS-PAGE. LDLR protein was detected with Coomassie brilliant blue R-250 stain. (C) Binding of PCSK9 to LDLR on ligand blots. LDLR protein $(2 \mu \mathrm{g})$ was transferred to nitrocellulose and incubated with $5 \mu \mathrm{g} / \mathrm{ml}$ of purified PCSK9 or no addition (NA). Bound PCSK9 was detected by immunoblot analysis as described in Methods. (D) The indicated amounts of purified LDLR were subjected to nonreduced SDS-PAGE, transferred to nitrocellulose, and incubated with $5 \mu \mathrm{g} / \mathrm{ml}$ of purified wild-type PCSK9, PCSK9(D374Y), or buffer control (no addition). Bound PCSK9 was detected by immunoblot analysis as described in Methods. pep., FLAG octapeptide.
In hepatocytes, the internalization of LDLRs is dependent upon functional autosomal recessive hypercholesterolemia (ARH), an adaptor protein that binds to the intracellular domain of the LDLR and to clathrin, thereby clustering the receptor in coated vesicles $(18,19)$. To determine whether ARH was similarly required for removal of LDLR from the cell surface, PCSK9 was added to the medium of primary hepatocytes isolated from 2 types of genetically engineered mice: those that express only the human LDLR $\left(L D L R^{b / h} A r b^{+/+}\right)(20)$ and those that express the human LDLR and lack ARH ( $\left.L D L R^{b / h} A r b^{-/-}\right)(21)$. In $L D L R^{b / h} A r b^{+/+}$hepatocytes, LDLRs were visualized on the cell surface and in intracellular vesicles concentrated in the cell periphery (Figure 6A, upper left). Incubation with PCSK9 eliminated the surface LDLRs, and the remaining LDLRs were concentrated in perinuclear vesicles (Figure 6A, upper right). In $L D L R^{b / h} A r b^{-/-}$hepatocytes, the LDLRs were visualized almost entirely on the cell surface (Figure 6A, lower left), and PCSK9 did not reduce the amount of surface LDLRs in these cells (Figure 6A, lower right).

To confirm that PCSK9 still associated with the cell in the absence of ARH, total lysates and cell-surface proteins were isolated for immunoblot analysis from $L D L R^{b / h} A r b^{+/+}$and $L D L R^{b / h} A r b^{-/-}$ primary hepatocytes incubated with increasing concentrations of PCSK9 (Figure 6B). The level of LDLR protein was reduced by exogenously added PCSK9 in a dose-dependent manner in both whole-cell extracts and on the cell surface in hepatocytes expressing ARH (lanes 1-3 and 9-11). In contrast, incubation of $L D L R^{b / h} \mathrm{Arb}^{-/-}$hepatocytes with PCSK9 did not alter LDLR protein levels in either fraction (lanes 4-6 and 12-14), despite similar lev- els of PCSK9 cell association (lanes 1-3 and 4-6). In the absence of ARH, significantly more PCSK9 was detected at the cell surface (lanes 13-14). No PCSK9 was detected in hepatocytes isolated from $L d l r^{--}$mice (lanes 7-8 and 15-16). PCSK9 had no effect on whole-cell or cell-surface transferrin receptors in hepatocytes, and cellular integrity was maintained during cell-surface biotinylation, as indicated by a lack of detection of intracellular actin among biotin-labeled proteins. These results demonstrate that PCSK9 can associate with the cell in the absence of ARH but that internalization is required for PCSK9 to reduce the cell-surface expression of LDLR protein. They also show that PCSK9 binding is largely dependent upon LDLR expression in mouse hepatocytes.

To test whether PCSK9 could function as a secreted protein in vivo, transgenic mice that express human PCSK9 in liver (TgPCSK9 mice) were produced. The transgene was under the control of an apoE promoter with a liver-specific enhancer (22). As shown in Figure 7A, transgenic overexpression of human PCSK9 eliminated LDLR protein expression in liver and caused a marked increase in plasma LDL cholesterol levels (Figure 7B). The increase in plasma LDL cholesterol was similar to that measured in $\mathrm{Ldl}^{-/-}$mice that lacked LDLRs in all tissues. The effects of transgenic PCSK9 overexpression are consistent with the results of previously published experiments in which adenoviral vectors were used to overexpress PCSK9 in mice $(3,8-10)$. The concentration of human PCSK9 in plasma from the transgenic mice as measured using the ELISA assay ranged from approximately 146 to $440 \mu \mathrm{g} / \mathrm{ml}$ (Table 1 , animals 9a, 10a, 11a, and 12a).

Although an apoE promoter fragment and a liver-specific enhancer were used to express the human PCSK9 cDNA in mice, 
A

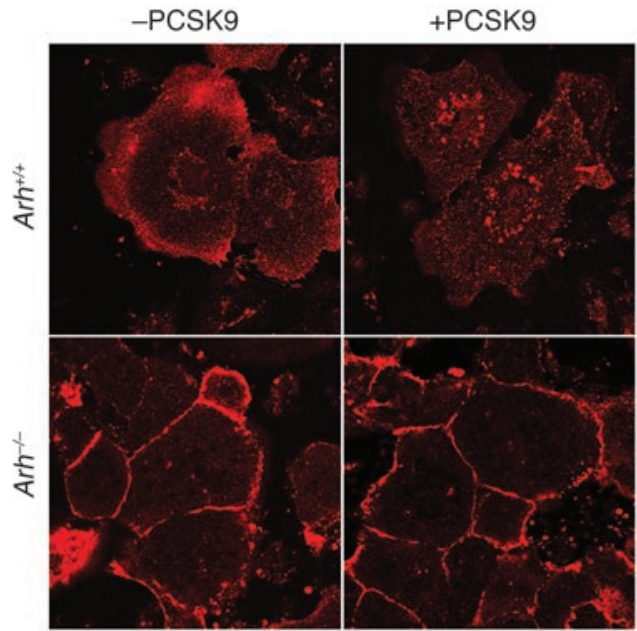

B

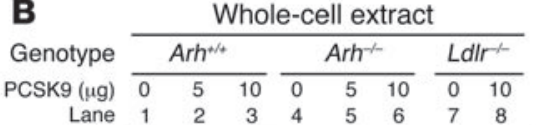

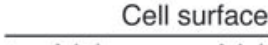

\begin{tabular}{|c|c|c|}
\hline $\mathrm{Arh}^{+/ *}$ & $\mathrm{Arh}^{-1}$ & Ldll $r^{\prime-}$ \\
\hline
\end{tabular}

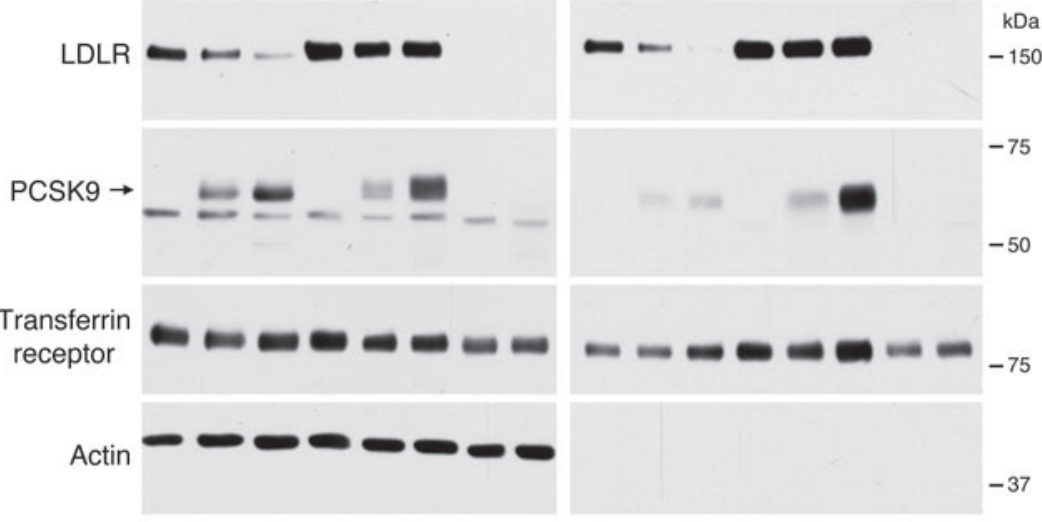

\section{Figure 6}

PCSK9-mediated degradation of the LDLR is dependent on $\mathrm{ARH}$. Immunofluorescence $(\mathbf{A})$ and immunoblot (B) analyses of the PCSK9-mediated changes of LDLR protein in mouse primary hepatocytes derived from $L D L R^{h / h} \mathrm{Arh}^{+/+}\left(\mathrm{Arh}^{+/+}\right)$or $L D L R^{h / h} \mathrm{Arh}^{-/-}\left(\mathrm{Arh}^{-/}\right)$mice. (A) Primary hepatocytes were incubated with no PCSK9 (-PCSK9) or with $5 \mu \mathrm{g} / \mathrm{ml}$ purified PCSK9 (+PCSK9) for 4 hours and processed for indirect immunofluorescence of LDLR using IgG-C7 and Alexa Fluor 563-conjugated secondary antibody, as described in Methods. Images were taken using a $63 \times 1.3$ objective using a confocal microscope (Leica TCS SP). Similar results were obtained in 2 independent experiments. (B) Immunoblot analysis of LDLR, FLAG-tagged PCSK9, transferrin receptor, and actin proteins in primary hepatocytes from mice of the indicated genotype incubated with varying amounts of PCSK9. the transgene mRNA was detected at low levels in other tissues, such as the adrenal gland (data not shown). This expression compromised analysis of the effect of plasma PCSK9 on LDLR protein expression in these extrahepatic tissues. Therefore, TgPCSK9 mice were parabiosed with wild-type mice to determine whether secreted PCSK9 derived from the transgenic mouse was able to decrease LDLR protein levels in liver when transferred via the shared circulation to the recipient wild-type mouse. For these studies, a liver biopsy was performed 1 week prior to parabiosis to obtain a liver sample for quantifying basal levels of LDLR protein expression. The mice were allowed to recover from the partial hepatectomy and then were parabiosed for 2 weeks to permit the development of shared circulation. To control for potential changes induced by surgery, wild-type mice were parabiosed with wild-type mice. TgPCSK9 mice manifest elevated plasma LDL cholesterol levels that could independently alter LDLR protein expression in the recipient wild-type mouse. To control for this effect, wild-type mice were parabiosed with $L d l r^{\prime-}$ mice, which have lipoprotein profiles similar to those of TgPCSK9 mice (Figure 7B).

As shown in Table 1, parabiosis itself slightly increased plasma total cholesterol levels in most mouse pairs. Plasma cholesterol concentrations in wild-type mice parabiosed with $\mathrm{Ldlr}^{-/-}$mice (pairs 5-8) were not significantly higher than those measured in the wild-type mouse parabiotic pairs (pairs 1-4). Concentrations of human PCSK9 in parabiotic TgPCSK9:wild-type pairs ranged from approximately 17 to $250 \mu \mathrm{g} / \mathrm{ml}$ (pairs 9-12), suggesting that the protein was transferred from the plasma of transgenic mice into the plasma of the parabiosed wild-type mouse with varying efficiencies. There also appeared to be a correlation between the plasma PCSK9 concentration and the degree of plasma cholesterol elevation of the recipient wild-type mouse. The wild-type mouse with the highest PCSK9 concentration (mouse 9b) had an approximately 10-fold increase in plasma total cholesterol, whereas the wild-type mouse with the lowest PCSK9 level (mouse 12b) had an approximately 2 -fold increase in plasma cholesterol.

To confirm that the observed plasma cholesterol changes were a result of alterations in LDLR protein levels, immunoblots of liver protein were performed in all parabiotic pairs before and 2 weeks after parabiosis (Figure 8). LDLR protein levels in livers of wildtype mice parabiosed with wild-type mice were not different before and after parabiosis (Figure 8A). Similarly, the LDLR protein levels in wild-type mice parabiosed with $\mathrm{Ldlr}^{--}$mice were not different after parabiosis, suggesting that the increased level of LDL cholesterol present in the knockout mouse did not alter LDLR expression in the wild-type mouse liver (Figure 8B). In contrast, the LDLR protein was essentially undetectable in livers of wild-type 
A

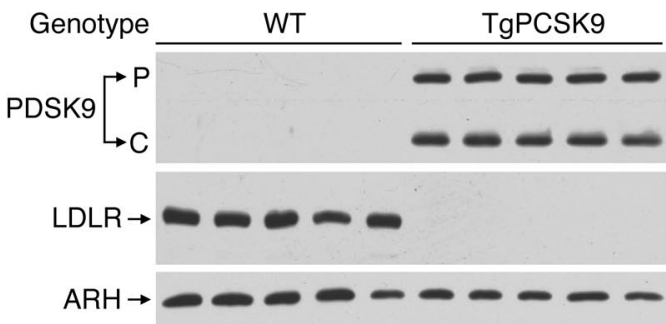

B

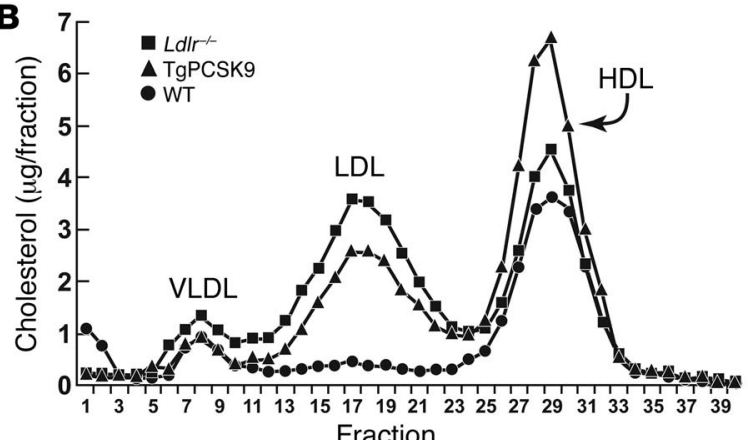

Fraction

mice after they were parabiosed with TgPCSK9 mice (Figure 8C), indicating that PCSK9 was active in mouse plasma.

To ensure that the reduction in LDLR protein levels measured in livers of the recipient wild-type mice parabiosed to the TgPCSK9 mice was due to posttranscriptional changes, mRNA levels of the LDLR and SREBP-2 were measured using real-time RT-PCR (Supplemental Table 1). Although individual mRNAs varied in amount from mouse to mouse, no consistent changes in the mRNAs encoding these genes were measured before and after parabiosis. Combined, the data suggested that secreted PCSK9 in plasma of mice reduced hepatic LDLR protein levels independent of changes in mRNA levels.

\section{Discussion}

In the current report, we demonstrate that endogenous PCSK9 is rapidly secreted from cells and that secreted PCSK9 destroys LDLRs when added to the medium of cultured HepG2 cells and mouse primary hepatocytes. The effective concentration of PCSK9 required to reduce the number of LDLRs in cultured cells was within the range of plasma concentrations measured in human plasma. The cell association and uptake of PCSK9 occurred via binding to the LDLR, and both proteins colocalized to a late endocytic/lysosomal compartment. The internalization of PCSK9 with the LDLR into an endosomal/lysosomal compartment was required for PCSK9 to reduce LDLR protein levels, since this activity was blocked in the absence of ARH. Finally, we show that PCSK9 was present in plasma of transgenic mice and that the secreted protein was active in destroying hepatic LDLRs.

Insights into the mechanism of secreted PCSK9's action were derived from studies in MEFs and mouse hepatocytes, which showed that LDLRs were required for the majority of PCSK9 to associate with the cell surface (Figure 4A and Figure 6B). These studies suggested that the LDLR and PCSK9 may directly interact, which was confirmed by coimmunoprecipitation and ligand blotting studies with the LDLR and PCSK9 (Figure 5). The coimmunoprecipitation studies with PCSK9(D374Y) and the LDLR suggested

\section{Figure 7}

Decreased amounts of hepatic LDLR protein and increased levels of plasma LDL cholesterol in transgenic PCSK9 mice. (A) Whole-cell lysates $(30 \mu \mathrm{g})$ from livers of 5 wild-type and $5 \mathrm{TgPCSK} 9$ mice were subjected to SDS-PAGE and immunoblot analyses of human PCSK9, mouse LDLR, and mouse ARH. (B) Plasma from the mice of each genotype described in $\mathbf{A}$ and from 5 age-matched male $\mathrm{Ldll}^{-/-}$mice was pooled and subjected to gel filtration by fast performance liquid chromatography. The concentration of total cholesterol in each fraction was measured as described in Methods.

that the interaction of the mutant protein with the LDLR was much more avid than that of the wild-type PCSK9 (Figure 5A). In addition, PCSK9(D374Y) was approximately 10-fold more efficient in destroying cell-surface LDLRs when added exogenously to HepG2 cells (Figure 3). Previous overexpression studies in McArdle-7777 rat hepatoma cells with PCSK9(D374Y) suggested that the mutant protein increased plasma LDL cholesterol levels through the overproduction of apoB (5). Similar conclusions were drawn from in vivo kinetic studies in humans that carry a different point mutation in PCSK9 (S127R) (23). The current studies with the D374Y mutant suggest an alternative mechanism that involves enhanced LDLR association and degradation. Whether the S127R and F216L point mutations in PCSK9 behave in a manner similar to PCSK9(D374Y) will be tested in future studies. Our observations that purified PCSK9(D374Y) added to cells reduced the number of LDLRs extend the results from a publication that appeared during the preparation of this manuscript demonstrat-

\section{Table 1}

Total plasma cholesterol and PCSK9 concentrations in pre- and postparabiotic pairs of wild-type, $\mathrm{Ldll}^{-}{ }^{-}$, and TgPCSK9 $(\mathrm{Tg})$ mice

\begin{tabular}{|c|c|c|c|c|}
\hline \multirow{2}{*}{$\begin{array}{l}\text { Parabiotic } \\
\text { pair }\end{array}$} & \multirow[t]{2}{*}{ Genotype } & \multirow{2}{*}{$\begin{array}{c}\text { Before parabiosis } \\
\text { Cholesterol } \\
\text { (mg/dl) }\end{array}$} & \multicolumn{2}{|c|}{ After parabiosis } \\
\hline & & & $\begin{array}{c}\text { Cholesterol } \\
\text { (mg/dl) }\end{array}$ & $\begin{array}{l}\text { hPCSK9 } \\
(\mu \mathrm{g} / \mathrm{ml})\end{array}$ \\
\hline $1 \mathrm{a}$ & WT & 54 & 79 & 0 \\
\hline $1 b$ & WT & 60 & 85 & 0 \\
\hline $2 a$ & WT & 67 & 91 & 0 \\
\hline $2 b$ & WT & 67 & 60 & 0 \\
\hline $3 a$ & WT & 54 & 85 & 0 \\
\hline $3 b$ & WT & 42 & 63 & 0 \\
\hline $4 a$ & WT & 51 & 81 & 0 \\
\hline $4 b$ & WT & 60 & 71 & 0 \\
\hline $5 a$ & $L d l r^{-1}$ & 178 & 288 & 0 \\
\hline $5 b$ & WT & 56 & 76 & 0 \\
\hline $6 a$ & $L d l r^{-1}$ & 182 & 270 & 0 \\
\hline $6 b$ & WT & 57 & 64 & 0 \\
\hline $7 a$ & $L d l r^{-1-}$ & 175 & 278 & 0 \\
\hline $7 b$ & WT & 53 & 82 & 0 \\
\hline $8 a$ & $L d l r^{-1}$ & 179 & 145 & 0 \\
\hline $8 b$ & WT & 68 & 101 & 0 \\
\hline $9 a$ & $\mathrm{Tg}$ & 142 & 365 & 440 \\
\hline $9 b$ & WT & 70 & 775 & 246 \\
\hline $10 \mathrm{a}$ & $\mathrm{Tg}$ & 132 & 350 & 198 \\
\hline $10 b$ & WT & 66 & 364 & 148 \\
\hline $11 a$ & $\mathrm{Tg}$ & 180 & 278 & 146 \\
\hline $11 b$ & WT & 61 & 233 & 80 \\
\hline $12 \mathrm{a}$ & $\mathrm{Tg}$ & 140 & 171 & 184 \\
\hline $12 b$ & WT & 61 & 131 & 17 \\
\hline
\end{tabular}




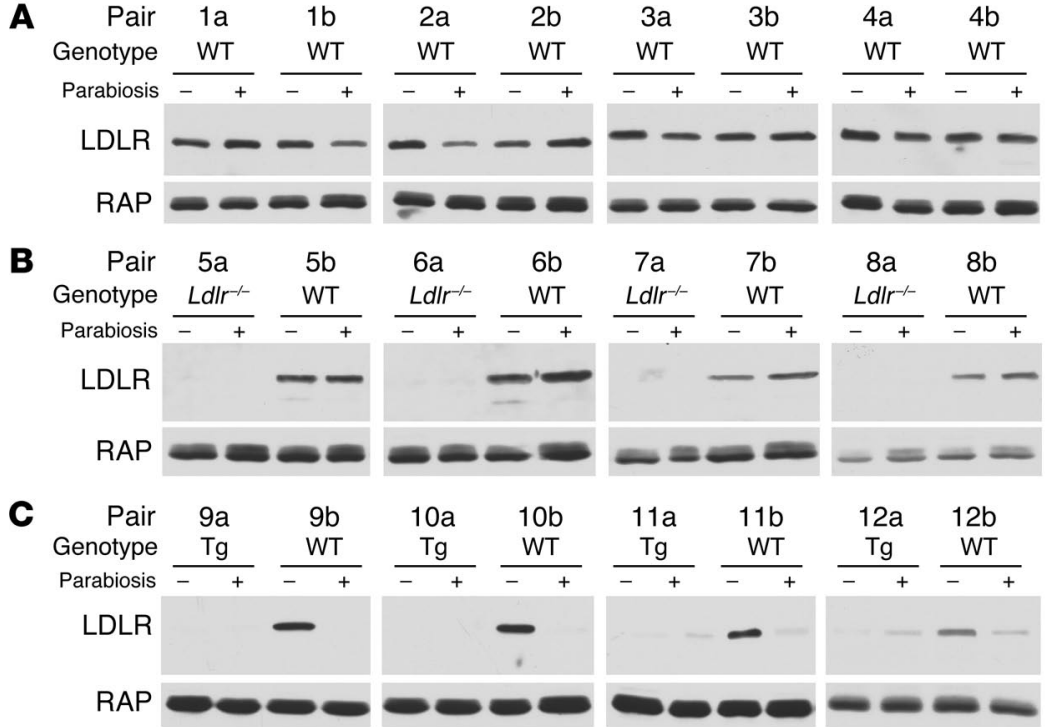

\section{Figure 8}

Amounts of LDLR protein in livers of parabiosed mice. Three combinations of mice were parabiosed for study: wild-type:wild-type (A); Ldlr-l-:wild-type (B); and TgPCSK9:wild-type (C). Prior to parabiosis, a liver sample $(<100 \mathrm{mg})$ via a partial hepatectomy and approximately $50 \mu \mathrm{l}$ of blood via the tail vein were obtained from each mouse. After a 7-day recovery period, 12- to 20-week-old female mice of the indicated genotype were parabiosed as described in Methods. After 2 weeks of parabiosis, the animals were killed, and blood and liver samples were collected for analysis. Liver membrane protein $(40 \mu \mathrm{g})$ obtained from each mouse before $(-)$ and after $(+)$ parabiosis was subjected to SDS-PAGE for immunoblot analyses of LDLR and RAP as a loading control (9). ing that conditioned medium containing PCSK9(D374Y) reduced the uptake of LDL (24).

A small amount of residual PCSK9 binding to LRP was detected in LDLR-deficient MEFs (Figure 4B). We have previously shown that hepatic LRP protein levels are not altered by the adenoviral overexpression of PCSK9 in mice (9), and LRP protein levels were not significantly different in livers of wild-type mice parabiosed with the TgPCSK9 mice (data not shown). Thus, although a small amount of PCSK9 may associate with LRP in vitro, it does not appear to significantly regulate LRP protein levels. The residual LRP-dependent cellular association of PCSK9 might be due to the sequence similarity between LRP and the LDLR. LRP contains 4 copies of the LDLR extracellular domain with similar charge distributions and may sufficiently resemble LDLR to support PCSK9 binding (25).

Recent studies by Maxwell et al. (26) showed that overexpression of PCSK9 in HepG2 cells induced the degradation of LDLR intracellularly in a post-ER compartment. These experiments also suggested that PCSK9-mediated reduction in the number of LDLRs was not attributable to the proteasome or lysosomal cysteine proteases. In addition, our previous studies using adenoviral overexpression of PCSK9 in mice demonstrated that high levels of PCSK9 expression in liver eliminated LDLR protein expression in wild-type and $\mathrm{Arb}^{-/-}$mice equally, indicating that functional ARH was not required for PCSK9 activity (9). This result differs from the data presented in Figure 6, which show that functional ARH is required for PCSK9 to destroy LDLRs when added exogenously to primary hepatocytes. Therefore, it is possible that PCSK9 overexpression may result in the association of PCSK9 with the LDLR within the cell and that this binding leads to intracellular LDLR protein degradation through the pathway described by Maxwell et al., whereas PCSK9's action at the cell surface is mediated by a distinct mechanism that is dependent upon ARH-mediated endocytosis.

Considered together, the available data now suggest that PCSK9 can function both extra- and intracellularly, but we do not know which pathway predominates under normal and/or pathologic conditions. Currently, all studies suggesting that the protein functions intracellularly have been performed using PCSK9 overexpression via a strong CMV promoter. Overexpression may permit association of PCSK9 and the LDLR in an intracellular compartment that does not occur physiologically. In the current studies, we were able to demonstrate that physiologically relevant concentrations of PCSK9 could significantly reduce the number of cell-surface LDLRs when added to HepG2 cells (Figures 2 and 3). Additional studies to determine the relative contribution of extracellular PCSK9 in the regulation of LDLR protein levels will require determining whether the infusion of PCSK9 into the circulation of PCSK9-knockout mice will decrease the number of LDLRs in liver and raise plasma cholesterol levels.

The results of the parabiosis studies shown in Figure 8 provide evidence that PCSK9 can function in plasma to destroy LDLRs in liver. The liver is the principal site of PCSK9 expression, and PCSK 9 is transcriptionally regulated by SREBP-2 (6). SREBP-2 regulates all genes required for cholesterol synthesis, and its activity is modulated by cellular sterol levels $(6,27)$. If the concentration of PCSK9 in plasma is primarily determined by the liver, then plasma PCSK9 levels may be a reflection of hepatic cholesterol stores, since PCSK9 transcription is reduced under conditions where SREBP-2 is suppressed, i.e., high cellular cholesterol concentrations. Such a regulatory event also raises the possibility that the liver indirectly regulates the amount of LDLRs in peripheral tissues by determining the amount of PCSK9 secreted into plasma. It is currently not known whether PCSK9 in plasma is capable of destroying LDLRs in tissues other than liver. This could not be directly addressed in the current studies, owing to the detection limits of the anti-LDLR antibody in tissues other than liver.

The genetic data from humans with loss-of-function mutations in PCSK9 combined with the studies in knockout mice that lack PCSK9 clearly indicate that inhibitors of the protease would be of therapeutic benefit for the treatment of hypercholesterolemia. Inasmuch as overexpression of the catalytically inactive form of PCSK9 in mice did not alter LDLR protein levels (9), an inhibitor of PCSK9's protease activity in the ER should be sufficient to block its ability to reduce LDLR protein levels. If PCSK9 functions as a secreted factor as suggested by the current data, then additional approaches to neutralize its activity, including the development of antibodies to block its interaction with the LDLR or inhibitors to block its action in plasma, can be explored for the treatment of hypercholesterolemia. 


\section{Methods}

Cultured cell experiments. HepG2, HEK 293 and MEF cells were cultured as described in Supplemental Methods.

Tissue culture medium. Medium A contained DMEM (cellgro; Mediatech Inc.) supplemented with $100 \mathrm{U} / \mathrm{ml}$ penicillin, $100 \mu \mathrm{g} / \mathrm{ml}$ streptomycin sulfate, and $1 \mathrm{~g} / \mathrm{l}$ glucose. Medium B contained medium A supplemented with $10 \%$ (vol/vol) FCS. Medium C contained medium A with 5\% (vol/vol) newborn calf lipoprotein-deficient serum (NCLPDS), $10 \mu \mathrm{M}$ sodium compactin, and $50 \mu \mathrm{M}$ sodium mevalonate. Medium D contained DMEM supplemented with $100 \mathrm{U} / \mathrm{ml}$ penicillin, $100 \mu \mathrm{g} / \mathrm{ml}$ streptomycin sulfate, and 4.3 $\mathrm{g} / \mathrm{l}$ glucose. Medium E contained medium D supplemented with $10 \%$ (vol/ vol) FCS. Medium F contained medium D supplemented with 5\% (vol/vol) NCLPDS, $10 \mu \mathrm{M}$ sodium compactin, and $50 \mu \mathrm{M}$ sodium mevalonate.

Purification of human wild-type and PCSK9(D374Y)-FLAG fusion proteins. HEK 293S cells stably expressing FLAG-tagged human PCSK9 or PCSK9(D374) were cultured in suspension without $\mathrm{CO}_{2}$ in IS GRO serum-free medium (Irvine Scientific) supplemented with 10\% FCS, $10 \mathrm{mM}$ L-glutamine, $100 \mathrm{U} / \mathrm{ml}$ penicillin, and $100 \mu \mathrm{g} / \mathrm{ml}$ streptomycin. PCSK9 was purified by anti-FLAG M2 affinity gel chromatography from $500 \mathrm{ml}$ of medium per the manufacturer's instructions, followed by size-exclusion chromatography on a Tricorn Superose 6 10/300 fast-performance liquid chromatography column (Amersham Biosciences). Fractions containing PCSK9 were concentrated approximately 5-fold using a Centricon filter (10 kDa-MW cut-off; Millipore). Protein purity was monitored by SDS-PAGE and Coomassie Brilliant Blue R-250 staining (Bio-Rad).

Antibodies. For the anti-human PCSK9 polyclonal antibody, the human PCSK9 amino acid sequence was analyzed using Protean software (Lasergene; DNAStar) for immunogenic regions. Amino acids 165-180 (RYRADEYQPPDGGSLV) and 220-240 (ASKCDSHGTHLAGVVSGRDAG) were synthesized, conjugated to keyhole-limpet hemocyanin using the Imject Maleimide Activated mcKLH kit (Pierce), and rabbits were injected with a mixture of the peptides (20 $\mu \mathrm{g}$ each) as described previously (28). IgG fractions from sera were purified using the ImmunoPure (A/G) IgG purification kit (Pierce).

Monoclonal antibodies that recognize human PCSK9 were generated by fusion of Sp2/mIL-6 (CRL-2016; ATCC) mouse myeloma cells with splenic B lymphocytes derived from a female BALB/c mouse injected with purified human PCSK9 protein (29). The resulting antibodies, 13D3 and 15A6, belong to IgG subclass 1 and recognize epitopes in the catalytic domain and the C-terminal region of PCSK9, respectively. Additional anitbodies used are described in Supplemental Methods.

Immunoblot analysis. Whole-cell lysate protein extracts were subjected to SDS-PAGE for immunoblot analysis as previously described (9). Biotinylation of cell-surface proteins of HepG2 cells was performed as previously described (30). Following biotinylation, whole-cell lysates were prepared, and protein concentration was determined. An aliquot of the whole-cell lysate was reserved, and the remainder was transferred to a fresh tube containing $50 \mu \mathrm{l}$ of Ultralink NeutrAvidin Protein Plus (Pierce) (1:1 slurry). After 18 hours of incubation with rotation at $4{ }^{\circ} \mathrm{C}$, beads were collected by centrifugation at $300 \mathrm{~g}$ for 5 minutes and washed 3 times with lysis buffer. Proteins were eluted from the beads by boiling in SDS-PAGE sample buffer and subjected to $8 \%$ SDS-PAGE and immunoblot analysis (9).

PCSK9 "sandwich" ELISA. LumiNunc Maxisorp white assay plates (Nunc) were coated with anti-human PCSK9 monoclonal antibody (IgG-13D3) diluted to $5 \mu \mathrm{g} / \mathrm{ml}$ in $100 \mu \mathrm{l}$ of buffer A $(20 \mathrm{mM}$ sodium phosphate $\mathrm{pH} 7.5,100 \mathrm{mM}$ sodium chloride) by incubation overnight at $4^{\circ} \mathrm{C}$. The assay plates were then washed with $350 \mu \mathrm{l}$ of PBS with Tween-20, pH 7.4 (PBSt) and blocked with $150 \mu \mathrm{l}$ of $0.5 \%$ BSA in buffer A for 1 hour at room temperature with shaking.

Sample preparation was carried out in a separate polypropylene microtiter sample plate. Standards were prepared in duplicate using dilutions of purified PCSK9 protein in buffer A plus $0.5 \%$ BSA. Plasma samples were prepared in triplicate by diluting $6 \mu \mathrm{l}$ of plasma in $84 \mu \mathrm{l}$ of buffer A plus $0.5 \%$ BSA. Thirty microliters of buffer B (buffer A, 0.5\% BSA, 7.2 M urea, and $0.68 \%$ Tween-20) was then added to each well, and the sample plate was incubated at $46^{\circ} \mathrm{C}$ for 30 minutes. Aliquots $(100 \mu \mathrm{l})$ were transferred to the assay plate and incubated for 2 hours at room temperature with shaking. The plate was then washed with PBSt and incubated for 1 hour at room temperature with $100 \mu \mathrm{l}$ of rabbit anti-human PCSK9 polyclonal antibody $(7.5 \mu \mathrm{g} / \mathrm{ml}$ in buffer A plus 0.5\% BSA). After washing with PBSt, $100 \mu \mathrm{l}$ of donkey anti-rabbit IgG plus HRP (GE Healthcare) diluted 1:10,000 was added for 1 hour at room temperature. After a final wash with PBSt, $100 \mu \mathrm{l}$ of SuperSignal ELISA Femto Substrate (Pierce) was added for 1 minute and luminescence quantified using a Dynex MLX microtiter plate luminometer (Dynex Technologies). To determine plasma PCSK9 concentrations, luminescence of the sample was compared with that generated in the standard curve with purified PCSK9. Assay validation experiments were conducted in accordance with current bioanalytical recommendations (31).

Human plasma was obtained from 72 volunteers ( 35 men and 37 women) after an overnight fast. Informed consent and blood samples were obtained for all participating subjects by institutional review board-approved protocols. The age of the individuals ranged from 21 to 56, and none were taking lipid-altering medications.

Pulse-chase analysis of PCSK9. HepG2 cells were cultured in medium C, and the pulse-chase was carried out as previously described (12). Immunoprecipitation of cell and medium extracts was performed using the polyclonal anti-PCSK9 antibody (32). Immunoprecipitates were eluted in SDS sample buffer and subjected to $8 \%$ SDS-PAGE. The gels were fixed and then immersed for 15 minutes in Amplify fluorogenic reagent (Amersham Biosciences), dried under vacuum, and exposed to film.

Coimmunoprecipitation of PCSK9 and the LDLR. HepG2 cells were cultured for 18 hours in medium C prior to treatment with $20 \mu \mathrm{g} / \mathrm{ml}$ or $2 \mu \mathrm{g} / \mathrm{ml}$ of purified PCSK9 or PCSK9(D374Y) for 1 hour in the presence of $0.1 \mathrm{mM}$ chloroquine. Pooled cell pellets from 6 dishes of cells were lysed in $2 \mathrm{ml}$ of buffer C (20 mM HEPES-KOH at pH 7.4, $150 \mathrm{mM} \mathrm{NaCl}, 1 \mathrm{mM} \mathrm{MgCl}$, and protease inhibitors) containing $1 \%$ (wt/vol) digitonin. Cell lysates were incubated with $50 \mu \mathrm{l}$ anti-FLAG M2 agarose (Sigma-Aldrich) to immunoprecipitate FLAG-tagged PCSK9 (for competition assays, $0.1 \mathrm{mg} / \mathrm{ml}$ of FLAG octapeptide was included); IgG-HL1 to immunoprecipitate LDLR; or IgG-2001, a mouse monoclonal antibody to an irrelevant antigen (33). Immunoprecipitates were washed 3 times with $500 \mu \mathrm{l}$ buffer $\mathrm{C}$ containing $0.1 \%$ (wt/vol) digitonin, eluted in SDS sample buffer by boiling for $5 \mathrm{~min}$ utes, and subjected to $8 \%$ SDS-PAGE.

Ligand blotting. Purified human LDLR extracellular domain (amino acids 1-699) was kindly provided by J. Deisenhofer (University of Texas Southwestern Medical Center) and was described previously (34). LDLR protein was resolved on nonreducing SDS-PAGE and transferred to nitrocellulose as described (35). LDLR protein was heated for 5 minutes at $96^{\circ} \mathrm{C}$ in sample buffer containing $2.5 \%$ (vol/vol) 2-mercaptoethanol for reducing conditions. Blots were blocked for 30 minutes in buffer D (50 mM Tris$\mathrm{Cl}$ at $\mathrm{pH}$ 7.4, $90 \mathrm{mM} \mathrm{NaCl}, 2 \mathrm{mM} \mathrm{CaCl}, 5 \%$ [wt/vol] BSA) and incubated sequentially for 60 minutes with gentle agitation in buffer $\mathrm{D}$ containing purified PCSK9 $(5 \mu \mathrm{g} / \mathrm{ml})$, the monoclonal anti-PCSK9 antibody IgG-15A6 $(5 \mu \mathrm{g} / \mathrm{ml})$, and HRP-conjugated goat anti-mouse IgG. PCSK9 and antibody incubations were followed by washes in buffer $\mathrm{E}(50 \mathrm{mM}$ Tris-Cl at $\mathrm{pH} 7.4$, $90 \mathrm{mM} \mathrm{NaCl}, 2 \mathrm{mM} \mathrm{CaCl}_{2}, 0.5 \%$ [wt/vol] BSA). Blots were visualized by immunoblotting as described above.

Immunofluorescence microscopy. Indirect immunofluorescence using antibodies that detect the LDLR, PCSK9, and CI-MPR in MEFs was performed as follows. MEFs cultured on glass coverslips to $50 \%$ confluence were fixed in $4 \%$ (wt/vol) paraformaldehyde in buffer $\mathrm{F}$ (PBS containing $2 \mathrm{mM} \mathrm{MgCl}_{2}$ ), 
quenched with $5 \mathrm{mM} \mathrm{NH}_{4} \mathrm{Cl}$ in buffer $\mathrm{F}$, permeabilized in buffer $\mathrm{F}$ containing $0.05 \%$ (wt/vol) Triton X-100 (Pierce) for 10 minutes at $-20^{\circ} \mathrm{C}$, and blocked for 30 minutes in buffer G (buffer F containing $1 \%$ [wt/vol] BSA). For double-label staining, cells were incubated sequentially with the monoclonal anti-PCSK9 antibody IgG-15A6 $(2.5 \mu \mathrm{g} / \mathrm{ml})$, Alexa Fluor 488-conjugated goat anti-mouse IgG $(0.6 \mu \mathrm{g} / \mathrm{ml})$, polyclonal LDLR antiserum Ab 4548 $(1: 1,000)$ or polyclonal CI-MPR IgG $(2 \mu \mathrm{g} / \mathrm{ml})$, and Alexa Fluor 555-conjugated goat anti-rabbit $\operatorname{IgG}(0.6 \mu \mathrm{g} / \mathrm{ml})$. Primary $\left(16\right.$ hours at $\left.4^{\circ} \mathrm{C}\right)$ and secondary $\left(1\right.$ hour at $\left.20^{\circ} \mathrm{C}\right)$ antibody incubations were followed by three 20-minute washes with buffer G. Coverslips were mounted in VECTASHIELD Mounting Medium (Vector Laboratories), and images were taken on a Zeiss confocal microscope (model LSM510-META). Indirect immunofluorescence for the LDLR in primary hepatocytes derived from $L D L R^{b / h} A r b^{+/+}$and $L D L R^{b / h} A r b^{-/-}$mice was carried out as previously described $(12,21)$.

TgPCSK9 mice. A cDNA encoding human PCSK9 was inserted into a pLiv-11 vector that contains the constitutive human apoE promoter and its hepatic control region (a gift from J. Taylor, Gladstone Institute of Cardiovascular Disease, San Francisco, California, USA) (22). Transgenic mice were generated by injecting linearized pLiv-11-hPCSK9 into the fertilized eggs as described previously (28).

Liver biopsy and parabiosis. One week prior to parabiosis, a liver biopsy was performed to obtain a liver sample for protein analysis. Mice were anesthetized with pentobarbitol, hair over the abdomen shaved, and skin cleaned with chlorhexidine. A $0.5-\mathrm{cm}$ transverse incision was made in the upper abdomen, and a small lobe of the liver $(\sim 50-100 \mathrm{mg})$ was tied off with a $5-0$ silk suture and excised. The liver sample was placed in liquid nitrogen for storage. The incision was closed in 2 layers using a 5-0 silk suture and wound clips. During sedation, $50 \mu$ l of blood was removed from the tail vein for plasma cholesterol measurements. The mice were allowed to recover from this surgery before the parabiosis was performed.

For parabiosis, mice were surgically joined following a previously published protocol (36), with slight modifications. Matching skin incisions were made from the shoulder to the hip joint of each mouse, and a $10-\mathrm{mm}$ incision was made in the muscles of the abdominal wall. The muscular incisions were joined using a 5-0 silk suture. The scapulae were attached by a 2-0 silk suture, and the skin was approximated using wound clips and 5-0 silk suture.

Three genetically distinct groups of female mice were studied: wild-type mice joined with wild-type mice; $L d l r^{-1}$ mice joined with wild-type mice; and TgPCSK9 mice joined with wild-type mice. Shared circulation was confirmed by injecting Evans blue into one of the parabiotic pairs prior to study. Mice were killed by isoflurane overdose 2 weeks after parabiosis, and blood and liver were harvested for analysis. Animal experiments were approved by the Institutional Animal Care and Research Advisory Committee at the University of Texas Southwestern Medical Center.

\section{Acknowledgments}

This work was supported by grants from the Perot Family Foundation and the NIH (HL-20948 and HL-38049). T.A. Lagace is supported by a fellowship from the Natural Sciences and Engineering Research Council of Canada. D.E. Curtis is supported by the UT Southwestern Physician Scientist Training Program. The authors wish to thank David W. Russell, Helen H. Hobbs, Jonathan Cohen, Joseph L. Goldstein, and Michael S. Brown for critical reading of the manuscript. Scott Clark, Amy Cox, Tuyet Dang, Linda Donnelly, Richard Gibson, Anh Pho, and Judy Sanchez provided excellent technical assistance.

Received for publication June 14, 2006, and accepted in revised form August 1, 2006.

Address correspondence to: Jay D. Horton, Departments of Molecular Genetics and Internal Medicine, University of Texas Southwestern Medical Center at Dallas, 5323 Harry Hines Blvd., Room L5-238, Dallas, Texas 75390-9046, USA. Phone: (214) 648-9677; Fax: (214) 648-8804; E-mail: jay.horton@utsouthwestern.edu.

Sahng Wook Park's present address is: Kwandong University College of Medicine, Department of Biochemistry, Kangneung, Republic of Korea.
1. Seidah, N.G., et al. 2003. The secretory proprotein convertase neural apoptosis-regulated convertase 1 (NARC-1): liver regeneration and neuronal differentiation. Proc. Natl. Acad. Sci. U. S. A. 100:928-933.

2. Naureckiene, S., et al. 2003. Functional characterization of Narc 1, a novel proteinase related to proteinase K. Arch. Biochem. Biophys. 420:55-67.

3. Benjannet, S., et al. 2004. NARC-1/PCSK9 and its natural mutants: zymogen cleavage and effects on the low density lipoprotein (LDL) receptor and LDL cholesterol. J. Biol. Chem. 279:48865-48875.

4. Abifadel, M., et al. 2003. Mutations in PCSK9 cause autosomal dominant hypercholesterolemia. Nat. Genet. 34:154-156.

5. Sun, X.-M., et al. 2005. Evidence for effect of mutant PCSK9 on apolipoprotein B secretion as the cause of unusually severe dominant hypercholesterolaemia. Hum. Mol. Genet. 14:1161-1169.

6. Horton, J.D., et al. 2003. Combined analysis of oligonucleotide microarray data from transgenic and knockout mice identifies direct SREBP target genes. Proc. Natl. Acad. Sci. U. S. A. 100:12027-12032.

7. Maxwell, K.N., Soccio, R.E., Duncan, E.M., Sehayek, E., and Breslow, J.L. 2003. Novel putative SREBP and LXR target genes identified by microarray analysis in liver of cholesterol-fed mice. J. Lipid Res. 44:2109-2119.

8. Maxwell, K.N., and Breslow, J.L. 2004. Adenoviralmediated expression of Pcsk9 in mice results in a low-density lipoprotein receptor knockout phenotype. Proc. Natl. Acad. Sci. U. S. A. 101:7100-7105.
9. Park, S.W., Moon, Y.-A., and Horton, J.D. 2004. Post-transcriptional regulation of low density lipoprotein receptor protein by proprotein convertase subtilisin/kexin type 9a in mouse liver. J. Biol. Chem. 279:50630-50638.

10. Lalanne, F., et al. 2005. Wild-type PCSK9 inhibits LDL clearance but does not affect apoB-containing lipoprotein production in mouse and cultured cells. J. Lipid Res. 46:1312-1319.

11. Cohen, J., et al. 2005. Low LDL cholesterol in individuals of African descent resulting from frequent nonsense mutations in PCSK9. Nat. Genet. 37:161-165.

12. Rashid, S., et al. 2005. Decreased plasma cholester$\mathrm{ol}$ and hypersensitivity to statins in mice lacking Pcsk9. Proc. Natl. Acad. Sci. U. S. A. 102:5374-5379.

13. Cohen, J.C., Boerwinkle, E., Moseley, T.H., and Hobbs, H.H. 2006. Sequence variations in PCSK9, low LDL, and protection against coronary heart disease. N. Engl. J. Med. 354:1264-1272.

14. Basak, A. 2005. Inhibitors of proprotein convertases. J. Mol. Med. 83:844-855.

15. Zhao, Z., et al. 2006. Molecular characterization of loss-of-function mutations in PCSK9 and identification of a compound heterozygote. Am.J. Hum. Genet. 79:514-523.

16. Naoumova, R.P., et al. 2005. Severe hypercholesterolemia in four British families with the D374Y mutation in the PCSK9 gene: long-term follow-up and treatment response. Arterioscler. Thromb. Vasc. Biol. 25:2654-2660.

17. Brown, W.J., Goodhouse, J., and Farquhar, M.G.
1986. Mannose-6-phosphate receptors for lysosomal enzymes cycle between the Golgi complex and endosomes. J. Cell Biol. 103:1235-1247.

18. Garcia, C.K., et al. 2001. Autosomal recessive hypercholesterolemia caused by mutations in a putative LDL receptor adaptor protein. Science. 292:1394-1398.

19. He, G., et al. 2002. ARH is a modular adaptor protein that interacts with the LDL receptor, clathrin, and AP-2. J. Biol. Chem. 277:44044-44049.

20. Knouff, C., Malloy, S., Wilder, J., Altenburg, M.K., and Maeda, N. 2001. Doubling expression of the low density lipoprotein receptor by truncation of the 3 '-untranslated region sequence ameliorates type III hyperlipoproteinemia in mice expressing the human apoe2 isoform. J. Biol. Chem. 276:3856-3862.

21. Jones, C., et al. 2003. Normal sorting but defective endocytosis of the low density lipoprotein receptor in mice with autosomal recessive hypercholesterolemia. J. Biol. Chem. 278:29024-29030.

22. Simonet, W.S., Bucay, N., Lauer, S.J., and Taylor, J.M. 1993. A far-downstream hepatocyte-specific control region directs expression of the linked human apolipoprotein E and C-I genes in transgenic mice. J. Biol. Chem. 268:8221-8229.

23. Ouguerram, K., et al. 2004. Apolipoprotein B100 metabolism in autosomal-dominant hypercholesterolemia related to mutations in PCSK9. Arterioscler. Thromb. Vasc. Biol. 24:1448-1453.

24. Cameron, J., et al. 2006. Effect of mutations in the PCSK9 gene on the cell surface LDL receptors. 
Hum. Mol. Genet. 15:1551-1558.

25. Herz, J., and Bock, H.H. 2002. Lipoprotein receptors in the nervous system. Annu. Rev. Biochem. 71:405-434.

26. Maxwell, K.N., Fisher, E.A., and Breslow, J.L. 2005. Overexpression of PCSK9 accelerates the degradation of the LDLR in a post-endoplasmic reticulum compartment. Proc. Natl. Acad. Sci. U. S. A. 102:2069-2074.

27. Brown, M.S., and Goldstein, J.L. 1997. The SREBP pathway: regulation of cholesterol metabolism by proteolysis of a membrane-bound transcription factor. Cell. 89:331-340.

28. Shimano, H., et al. 1996. Overproduction of cholesterol and fatty acids causes massive liver enlargement in transgenic mice expressing truncated SREBP-1a. J. Clin. Invest. 98:1575-1584.
29. Herz, J., Kowal, R.C., Ho, Y.K., Brown, M.S., and Goldstein, J.L. 1990. Low density lipoprotein receptor-related protein mediates endocytosis of monoclonal antibodies in cultured cells and rabbit liver. J. Biol. Chem. 265:21355-21362.

30. Hanwell, D., Ishikawa, T., Saleki, R., and Rotin, D. 2002. Trafficking and cell surface stability of the epithelial $\mathrm{Na}+$ channel expressed in epithelial Madin-Darby canine kidney cells. J. Biol. Chem. 277:9772-9779.

31. DeSilva, B., et al. 2003. Recommendations for the bioanalytical method validation of ligand-binding assays to support pharmacokinetic assessments of macromolecules. Pharm. Res. 20:1885-1900.

32. Sakai, J., et al. 1997. Identification of complexes between the COOH-terminal domains of sterol regulatory element-binding proteins (SREBPs) and
SREBP cleavage-activating protein. J. Biol. Chem. 272:20213-20221.

33. Beisiegel, U., Schneider, W.J., Brown, M.S., and Goldstein, J.L. 1982. Immunoblot analysis of low density lipoprotein receptors in fibroblasts from subjects with familial hypercholesterolemia. J. Biol. Chem. 257:13150-13156.

34. Rudenko, G., et al. 2002. Structure of the LDL receptor extracellular domain at endosomal $\mathrm{pH}$. Science. 298:2353-2358.

35. Daniel, T.O., Schneider, W.J., Goldstein, J.L., and Brown, M.S. 1983. Visualization of lipoprotein receptors by ligand blotting. J. Biol. Chem. 258:4606-4611. 36. Wright, D.E., Wagers, A.J., Gulati, A.P., Johnson, F.L., and Weissman, I.L. 2001. Physiological migration of hematopoietic stem and progenitor cells. Science. 294:1933-1936. 